Authors

Meredith Oatley, RN, GradCert (Cancer Nursing), MN (NP)

Nurse Practitioner Oncology

Royal North Shore Hospital

Reserve Road, St Leonards, Sydney, New South Wales, 2065

Australia

Email: meredith.oatley@health.nsw.gov.au

$\mathrm{T}:+61402158169$

Margaret Fry RN, NP, BaSc (Nursing), MEd, PhD

Director Research and Practice Development NSLHD

Professor of Nursing, University of Technology Sydney

Adjunct Professor of Nursing, University of Sydney

Royal North Shore Hospital

Reserve Road, St Leonards, Sydney New South Wales, 2065

Australia

Email: margaret.fry@uts.edu.au

T: +61425313391

ORCID 0000-0003-1265-7096 


\section{A NURSE PRACTITIONER LED MODEL OF CARE IMPROVES ACCESS, EARLY ASSESSMENT AND INTEGRATION OF ONCOLOGY SERVICES: AN EVALUATION STUDY}

Purpose: In Australia, the number of cancer cases has doubled since 1991 and is the second most common cause of death [1]. Chemotherapy, a common treatment, is known to cause distressing symptoms that often lead to a person presenting to an emergency department (ED).

The aim of this study was to investigate whether a Nurse Practitioner (NP) led model of care could improve cancer service integration and reduce hospital presentations.

Methods: This was a evaluation study for a new model of care that included i) telephone helpline; ii) urgent assessment clinic; iii) rapid day treatment consultation service.

Results: The utilisation rate was 337 telephone calls involving 157 patients in the 7-month pilot. The most common reason for calling the helpline was for symptom management $(n=173: 51 \%)$, followed by education regarding treatment $(n=61: 18 \%)$. As a result $49 \%$ $(n=165)$ of callers were given advice, information or education, $22 \%(n=74)$ were referred on to other healthcare providers and $11 \%$ (38) were admitted to hospital. Of the 38 admitted patients, 9 were admitted directly from the urgent NP-led clinic bypassing the ED.

Conclusions: The implementation of the NP-led model of care has reduced ED presentations, optimised symptom management, and streamlined patient telephone enquiries using validated clinical assessment tools [2] within cancer services. The telephone helpline was available for the broader local health district community and was actively utilised. Patient surveys were overwhelmingly positive. The model of care has improved symptom management for patients and reduced ED workload and presentation rates.

Keywords: Oncology, Models of care, Survey, Day treatment unit 
Submission requirements:

Title: A Nurse Practitioner Led Model of Care improves access, early assessment and integration of oncology services: an evaluation study

Journal: Supportive Care In Cancer

Authors: Meredith Oatley, Margaret Fry

Meredith.oatley@health.nsw.gov.au

Affiliations:

Northern Sydney Local Health District, Royal North Shore Hospital,

New South Wales, Australia

University of Technology Sydney NSW Australia 


\section{A NURSE PRACTITIONER LED MODEL OF CARE IMPROVES ACCESS, EARLY ASSESSMENT AND INTEGRATION OF ONCOLOGY SERVICES: AN EVALUATION STUDY}

\section{INTRODUCTION}

Emergency admissions are increasing globally creating pressure on hospital systems and health budgets [1]. Internationally, to support people with cancer receiving chemotherapy and experiencing treatment side effects a range of strategies has been implemented. Across the UK, Canada, USA and Europe telephone helplines have been implemented to support clinical need and reduce emergency presentations and optimise timely and appropriate integrative care [1-5]. The success of this strategy is most notable in the UK where a dedicated telephone triage service has been implemented nationally [2].

However, across Australia, patients that experience treatment related side effects are often required to manage these symptoms at home with limited support. In Australia, for people receiving cancer treatments, the health options for symptom management usually involve presenting to a primary care physician, ED, or telephoning hospital cancer services. However, hospital telephone cancer services are ad hoc or non-existent $[6,7]$. Further, there is little evidence of telephone helpline service availability, structure, staff responsible for providing the service, or evaluation [7]. More importantly, and unlike international telephone helpline services, there is no extant literature of the clinicians that provide telephone triage, educational requirements and or expertise needed to undertake the role.

A recent study highlighted that many people present to a tertiary referral ED for the management of treatment side effects and commonly during business hours [7]. These findings suggest that there were limited support services available for patients undergoing cancer treatment and that patients were often confused about health choices. Therefore, the aim of the study was to investigate a new Nurse Practitioner (NP) led model of care that included a i) oncology telephone helpline for patients, families and clinicians; ii) urgent assessment clinic; and iii) a rapid consultation service for the Day Treatment Unit.

\section{METHODS}

The evaluation study sought to examine the new NP-led intervention. The study was conducted over seven months (1st March 2018 to 30th September 2018). 


\section{SITE AND SAMPLE}

The study was conducted in a metropolitan tertiary referral hospital within the state of New South Wales, Australia. The hospital has 600 beds and provides inpatient and ambulatory cancer services.

The sample involved adult (over 16 years) medical oncology patients receiving active treatment. In addition, carers and or family members, tertiary hospital clinicians' local primary health care providers (general practice physicians, dentists, community nurses, community palliative care services) were invited to use the helpline service.

All new patients receiving chemotherapy were educated about the telephone helpline. Patients were provided with a laminated telephone helpline alert card with the 1800 telephone helpline number.

\section{INTERVENTION}

The intervention involved a NP-led oncology telephone helpline service, urgent assessment clinic, and a consultation service for the Day Treatment Unit (DTU). The model of care was translated from available international literature [2-5]. Importantly, the intervention provided for the first time in New South Wales a systematic approach to the telephone triaging of medical cancer patients and families. All patient episodes were documented in the electronic medical record.

Stakeholder engagement for the project was sort through the delivery of formal education sessions in the hospital ED, the community palliative care hospitals and the hospital cancer centre. The educational sessions were for clinical, executive and administrative staff. Further stakeholder information forums were given to individual clinical specialists within the departments of radiation oncology, palliative care and medical oncology. Patient education forums were also given weekly in the new patient orientation program at the cancer centre. These information sessions were to principally promote and inform clinicians and patients about the helpline service and how it could be utilised. The strategy of health promotion was strengthened through the distribution of health information pamphlets for patients and staff within the hospital and the broader health district.

\section{TELEPHONE HELPLINE}

The telephone helpline was implemented and managed by a newly appointed oncology NP and operated Monday to Friday (8.00am to 4.30pmhealth enquiries outside of these hours there was an automated answering service with a message bank, with instructions to attend the ED, a general practitioner or call ambulance services. Patients were contacted the next 
business day if a message was left on this service. The UK validated telephone triage tool [8] was adopted for the implementation once permission was obtained to use the Triage Rapid Assessment and Access Tool Kit. Telephone assessment is completed using the triage tool and patients' are allocated one of three triage codes 1) Advice and reassurance (green), 2) 24 hour follow up (orange) and 3) Assess (red). The triage tool collected patient demographics, reason for calling, medical history and medications, and signs and symptoms. The tool provided for a systematic approach to prioritise patient need, assessment, management, and referral.

\section{URGENT ASSESSMENT CLINIC}

The new model of care involved an NP led urgent assessment clinic, which would review all patients referred from the helpline. In addition, medical inpatient teams could refer patients for review and patient could self-select to present to the clinic. All code red patients were referred by the NP to the ED. All patients assessed as code orange were reviewed in the urgent assessment clinic within 24 hours. The NP-led urgent assessment clinic operated Monday to Friday (8.00am to $4.30 \mathrm{pm}$ ) and would, for the first time in NSW, provide for integrated care through timely patient assessment, management and or referral to appropriate primary or acute oncology medical services.

\section{DAY TREATMENT UNIT}

The new model of care involved a consultation service to the DTU. Nurses or medical staff could request a patient consult for anyone undergoing immediate treatment. The NP would review cases and manage clinical treatment issues.

The model of care removed the ad hoc health district system processes, poor access to ambulatory clinics, DTU patient review delays and inconsistent management of hospital telephone enquiries. Poorly managed telephone enquiries often led to suboptimal patient care, poor information transfer, ED presentations and a lack of integrative care and consistency in care practices.

\section{DATA COLLECTION}

Data collection included the: UK triage collection tool, patient electronic medical record and a paper patient and family satisfaction survey. The UK triage tool collected: date and time, caller demographics and history, diagnosis and treatment, assessment criteria, medications, and outcomes. Electronic medical record data included: patient demographic (age, medical record number, gender, postcode) and clinical information including: diagnosis, time, caller, past medical history, medical specialist, reason for call, active treatment, treatment protocol, 
part of a clinical trial, date of last treatment, temperature, central line in situ, plan outcome and referral.

The patient survey was conducted at the completion of the study and contained 28 questions. Questions focused on advice given; the quality of the telephone advice; what alternative actions the patient would have taken in the absence of a helpline service; overall experience; satisfaction level and outcomes. All patients that used the telephone helpline were invited to respond and place the anonymous survey in a sealed box located in the cancer centre.

Quantitative data collected was analysed using IBM SPSS v.22. Descriptive statistics (frequency and percentages) were used to provide a summary of the data. Data that was normally distributed were analysed using mean and standard deviation. Non-parametric testing was conducted for skewed data (median, interquartile ranges). Depending on the distribution of data Pearsons Chi-Square (X2) test or student t-test was used to measure association. Statistical analysis, including descriptive, comparative and correlational analysis was completed using the IBM SPSS program (IBM SPSS v.22, Chicago IL USA).

\section{ETHICAL APPROVAL}

Ethical approval was obtained from a local Lead Human Research Ethics Committee. Data were stored in password protected files or stored in a locked cabinet accessible by the chief investigators. Patient details were de-identified to ensure confidentiality, beneficence and privacy.

\section{RESULTS}

The telephone helpline provided 337 consultations and involved patients $(\mathrm{n}=203 ; 60.2 \%)$, families/carers $(n=110 ; 33.0 \%)$ and clinicians $(n=24 ; 7.1 \%)$. There were, of the telephone callers, 135 males $(40 \%)$ and 202 females (60\%) (Table 1). Often multiple consultations occurred for individual patients with 157 individual cancer patients reviewed. The majority of patients that called the helpline had a diagnosis of breast cancer $(n=79)$, colorectal cancer $(n=51)$ or pancreatic cancer $(n=33)$.

Of the 157 individual patients, the average patient age was 63.2 years (SD 14.0years) with men slightly older (65years, 13.5years) than females (61 years; 14.0years) (Table 2). The most common reason for calling the helpline was for symptom management $(n=173 ; 51 \%$ ), followed by education regarding symptom treatment $(n=61 ; 18 \%)$. The main reported symptoms were pain $(n=49 ; 15 \%)$, fatigue $(n=27 ; 8 \%)$, nausea $(n=22 ; 7 \%)$ or dyspnea / shortness of breath $(n=22 ; 7 \%)$. Outcomes of the helpline service identified that $51 \%(n=173)$ of callers were given symptom advice followed by health care education $(n=61,22 \%)$. 
There was no statistical difference $(\mathrm{p}>0.05)$ for day of the week of the telephone calls, although there was a trend for a higher proportion of calls on Tuesdays $(n=75)$. A higher number of telephone calls were received during the first four hours of the day between 08:00 - 12:00 (Table 2). The average telephone consultation lasted 16 minutes (SD 9minutes).

Of the three assessment categories, the majority of calls $(n=189 ; 56 \%)$ were for category 'advise and reassurance' (green) (Table 3). Telephone helpline consultations required 22\% $(n=74)$ of patients to be reviewed and or referred on to medical oncology specialists. Of the 74 patients, $38(11 \%)$ required immediate review and hospital admission. Of the patients that required hospital admission $9(23.7 \%)$ were admitted directly from the NP-led urgent assessment clinic bypassing the ED.

The NP-led urgent assessment clinic managed 297 patients. Of these 297 patients, 38 (24.2\%) were referred from the helpline for immediate review. All triaged code orange patients $(n=117)$ were reviewed in the clinic within 24 hours. Patients $(n=59)$ aware of the clinic could self-refer for assessment by the NP. Clinic patients $(n=121)$ were also referred by in-patient medical teams. Patients that presented to the NP urgent assessment clinic were reported as an avoidable ED admission which translated to an $11 \%$ reduction in ED presentations.

Patient consultations for the DTU occurred on average four times per day. NP activities involved assessment and review $(\mathrm{n}=422)$; prescribing to support symptom management $(n=212)$; prescribing of intravenous fluid $(n=120)$; consenting for blood products $(n=22)$; pathology review $(n=482)$; neurological assessment $(n=24)$; infusion reaction management $(n=24)$; and responding to patient deterioration $(n=5)$.

The helpline patient and family survey response rate was $30 \%(n=47)$. The majority of respondents were female $(\mathrm{n}=26 ; 55.3 \%)$ with an average age of 59.7 years (SD13.0years). The average age for all respondents was 64 years (SD 13.2 years). The helpline was accessed by $34(72.3 \%)$ patients more than twice and $11(23.4 \%)$ used the helpline more than five times during their treatment.

The majority of patients reported that they would have called hospital services $(n=20 ; 45.5 \%)$ or attended the $\operatorname{ED}(n=10 ; 23.8 \%)$ in the absence of the helpline. Eight patients $(18.2 \%)$ reported that they would have done nothing about their symptoms. Of the 47 respondents, 44 $(96.0 \%)$ reported the telephone helpline was easy to access.

The survey respondents reported being "very satisfied" with the telephone helpline service, and 43 (91.5\%) reported instructions were clear (4 missing data sets). Forty (89.0\%) patients reported that the helpline service increased the quality of care offered within the health setting. Of the individual cases accessing the telephone helpline, $13.6 \%(n=46)$ had died within the study period. 


\section{DISCUSSION}

The evaluation demonstrated that the NP-led model of care supported the delivery of safe, appropriate and timely care for adult oncology patients. The model of care improved choice for patients and families facilitated integrated care and assisted to reduce time delays in access, review and assessment by hospital oncology experts. The model of care has demonstrated that timely assessment and access to an expert oncology clinician has mitigated more serious outcomes for patients and reduced ED and hospital admissions.

Telephone helplines have been shown to improve access, provide an alternative pathway to presenting to an ED, and increase patient and family satisfaction and choice [8, 9]. The model of care implemented was innovative as the telephone helpline was also available to support general physicians, community nurses, palliative care services and community based health professional. Advice focused on treatment symptoms and side effects of cancer treatments and supported an integrative approach between community and acute services. This evaluation demonstrated that a telephone helpline provided for early referral, review and assessment of and for patients and is feasible as a NP-led model of care.

International evidence recommends that comprehensive cancer services need to have structured telephone helplines to expedite the assessment, management and referral of patients experiencing treatment related toxicities $[3,9,10]$. In NSW there was no one point of contact where patients could telephone and receive specialist advice, referral or treatment. Consumers experiencing cancer require a support to enable decision making when faced with treatment side effects or problems. This model of care enabled effective and timely communication that was focused on patient safety and support [11].

The evaluation has shown the model of care to be feasible and practical with potential reach and scale. The intervention could extend to different diagnostic and age groups, geographical locations and clinical settings (including Residential Aged Care Facilities; community care; and public-private partnerships). Importantly, this study translated UK oncology resources and tools, which could easily be adopted across Australia enabling a national approach to support and manage people experiencing cancer treatment related symptoms.

The utility of an NP-led urgent assessment clinic demonstrated a high volume of patient encounters while supporting patient safety, choice and a structured integrative pathway between community and acute services. Specifically, the clinic enabled a more timely care response for those patients managing treatment symptoms in the community. Patients' choice was expanded with implementation of clinic enabling patients to self-refer. Similar 
interventions operate throughout the USA and support the development of these NP-led services $[12,13]$.

The new model of care also enabled a more timely care response for those patients undergoing active cancer treats in the DTU. The NP provided a consultation service for the DTU, which resulted in the timely clinical review of patients. Nursing and medical staff were able to request immediate review of patients reducing assessment and medication delays.

The new model of care has provided an alternative pathway for patients and carers to better support them to navigate health issues, access, equity and self-management. Currently in NSW there was no one point of contact where patients could telephone and receive specialist advice. Yet decisions about what to do when faced with treatment side effects requires a structured, systematic and supportive approach. This intervention has improved timely support, patient referrals into appropriate services and provided greater integration of care between acute and community services. A lack of alternative pathways often results in cancer patients presenting to EDs who would be better-managed by oncology experts [3, 7, 14].

The NP-led model of care project was awarded, in the 'Transforming the Patient Experience' category, a 2019 New South Wales Ministry of Health Award. Further, the impact evidence presented to the Local health District led to the project receiving ongoing permanent funding to sustain the service within the hospital and health district. The NP model of care remains fully functional operating Monday - Friday (8.00am to $4.30 \mathrm{pm}$ ) and has employed a full time equivalent clinical nurse consultant in addition to the NP. Further, analysis of service utility will be explored to evaluate the potential for an afterhours service.

\section{LIMITATIONS}

There are a number of limitations with this study that should be considered. A convenience sample was used for the patient survey and relied on self-reporting, which may not be representative of oncology patients throughout Australia. The study was conducted in a single tertiary referral oncology hospital, and the findings may not have relevance to other hospitals or oncology settings. Due to resources, staff evaluation surveys were not conducted which may have provided different insights into the benefit, utility and appropriateness of the model of care.

Sample bias may be present as the patients accessing the model of care may be different to those that chose not to utilize the telephone helpline. Hence the findings may not reflect the perceptions of oncology patients and clinicians in other health settings. The telephone helpline was only available during business hours (8.00am to $4.30 \mathrm{pm})$. Afterhours calls were recorded on the answering service and patients were contacted the next working day. The 
findings therefore may not be generalizable to oncology patients experiencing treatment side effects after hours.

\section{CONCLUSION}

The evaluation has demonstrated that a NP led model of care assisted to improve the delivery of safe, appropriate and timely care for medical oncology adult patients. Patients and families experienced greater choice, facilitated integrative care, support in navigating preferences and expectations, and reduced time delays to access and assessment by hospital oncology experts. The DTU consultation service provided by the NP also improved timely review and assessment of people experiencing immediate treatment side effects that if left unchecked could potentially lead to more serious patient related outcomes.

This study translated and implemented UK triage oncology resources and tools, which easily accommodated the Australian health care context. Further research is needed to explore the opportunities for national translation to better support patients experiencing treatment side effects across Australia that are geographically challenged. The model of care enhanced the quality and safety of care for people experiencing cancer treatment side effects brought about timely multidisciplinary and interprofessional communication, improved medical record documentation, and a reduction in ED presentations.

Financial disclosure

There are no conflicts of interest to declare for authors MO and MF. There was no conflict of interest, activities or potential for influencing this work. The funding of this study did not influence the collection or analysis of this study.

We wish to disclose that funding was received from the Ministry of Health Nursing and Midwifery Officer of New South Wales Australia. There was no conflict of interest, activities or potential for influencing this work by the funders. The grant organisation had no financial interest or role in the design, conduct or analysis or the writing up of this study.

\section{Conflicts of interest}

There are no conflicts of interest to declare for authors MO and MF. There was no conflict of interest, activities or potential for influencing this work. The writing of the manuscript was undertaken by the two authors only. The authors whose names are listed have NO affiliations with or involvement in any of the organization or entity that has provided funding for the 
study. The named authors have full control of the primary data. However, in line with ethics requirements in Australia the authors are unable to provide data for review. 


\section{REFERENCES}

1. Chen, $\mathrm{H}$., et al., Emergency adissions and subsequent inpatient care through an emergency oncology service at a tertiary cancer centre: service users experiences and views. Supportive Care in Cancer, 2018. 27: p. 451-460.

2. Jones, P., Oncology/Haematology 24 Hour Triage Rapid Assessment and Access Toolkit Australian Version in United Kingdome Oncology Society 2018, UKONs: United Kingdom p. 27.

3. Stacey, D., et al. Development and evaluation of evidence-informed clinical nursing protocols for remote assessment, triage and support of cancer treatmentinduced symptoms. Nursing research and practice, 2013. 2013.

4. Ruegg, T., A Nurse Pracitioner-Led Urgent Care Center: Meeting the Needs of the Patient with Cancer. Clinical Journal of Oncology Nursing, 2013. 17(4): p. E52E57.

5. Mayer, D.K., et al., Why do patients with cancer visit emergency departments? Results of a 2008 population study in North Carolina. J Clin Oncol, 2011. 29(19): p. 2683-8.

6. McKenzie, H., et al., Chemotherapy outpatients' unplanned presentations to hospital: a retrospective study. Support Care Cancer, 2011. 19(7): p. 963-9.

7. Oatley, M., M. Fry, and L. Mullen, A Cross-sectional Study of the Clinical Characteristics of Cancer Patients Presenting to one Tertiary Referral Emergency Department. International Emergency Nursing, 2016. 24: p. 35-38.

8. Jones, P., Oncology/Haematology 24 Hour Triage Rapid Assessment and Access Tool Kit, in Oncology/Haematology 24 Hour Triage, C. Hughes, Editor. 2010, UKONS/Greater Midlands Cancer Network: United Kingdom. p. 1-49.

9. Towle, E., Telephone Triage in Today's Oncology Practice. American Society of Clinical Oncology, 2009. March: p. 1.

10. Stacey, D. and L. Fawcett, Telephone triage: an important role for oncology nurses. Canadian oncology nursing journal $=$ Revue canadienne de nursing oncologique, 1997. 7(3): p. 178-9.

11. Australian Council of Healthcare Standards, NSQHS Australian Comission on Safety and Quality in health Care, Editor. 2017, Australian Government.

12. Weinick, R.M., R.M. Burns, and A. Mehrotra, Many emergency department visits could be managed at urgent care centers and retail clinics. Health Aff (Millwood), 2010. 29(9): p. 1630-6.

13. Barbera, L., C. Taylor, and D. Dudgeon, Why do patients with cancer visit the emergency department near the end of life? Cmaj, 2010. 182(6): p. 563-8.

14. Bureau of Health Information, Spotlight on Measurement: Emergency department utilisation by people with cancer, N. Government, Editor. 2014, State Health publication: Sydney, NSW. p. 8. 
